\title{
Case reports and the Journal: an update for authors and readers
}

\author{
Donald R. Miller, MD • Francois Donati, MD, PhD
}

Published online: 9 April 2010

(C) Canadian Anesthesiologists' Society 2010

As with a number of scientific medical specialty journals, we have continued to receive many more case reports/case series (hereafter referred to in this article as "case reports") over the years than we have been able to publish. We recognize that clinicians are interested in case reports that are well-written, appropriately referenced, and adequately detailed, as such reports can add to the body of clinical knowledge by reporting rare or unusual clinical events that cannot be studied or evaluated in a clinical trial setting. Also, we appreciate that some medical journals have limited the number of case reports or have stopped publishing case reports altogether due to changes in editorial policy and/or a down-drafting effect on a journal's impact factor (mean journal citation frequency). However, considering our largely clinical readership, we continue to value, as part of our editorial content, appropriately balanced case reports from recent clinical experiences involving anesthesia, perioperative medicine, pain management, and critical care. At the same time, we have identified a number of recurrent issues that often result in many submitted case reports being rejected even before external review. In view of these recurrent issues, we prepared this editorial to provide an overview for potential authors and readers of the Journal regarding our requirements for considering a case report for publication and our editorial perspectives concerning content that may be suitable for publication.

D. R. Miller, MD $(\bowtie)$

Department of Anesthesiology, The Ottawa Hospital, University of Ottawa, General Campus, CCW 1409, 501 Smyth Road, Ottawa, ON K1H 8L6, Canada

e-mail: cja_office@cas.ca

F. Donati, $\mathrm{MD}, \mathrm{PhD}$

Maisonneuve-Rosemont Hospital, University of Montreal, Montreal, QC, Canada
A basic pre-requisite of any case report submitted to the Journal is that it must conform to the requirements of the particular type of article; these details are posted online in the Instructions for Authors (available at: http://cja.edmgr. com). In addition to adhering to the general principles of article preparation specific to the Journal, special attention should be directed towards adhering to the word count limit (maximum 2,000 words excluding the abstract), good image quality, proper legends, and a limited number of relevant references (10-25). We especially encourage authors to ensure that they use sound formatting, grammar, syntax, spelling, and scientific and clinical terminology in their articles. While we do not expect all authors who write and submit case reports to be highly published or clinicianscientists, any author submitting a case report should be aware that incomplete or improper article preparation may provide grounds for rejection before an external review is even considered.

Authorship should be limited to those who have had a direct role in the writing and interpretation of the report. A long list of authors (we receive many case reports listing more than four authors) may be inappropriate for most case reports. When more than two or three clinicians were involved with the clinical care of the reported case, but not with the actual writing, consideration may be given to acknowledging these individuals separately. All listed authors should have made material contributions to the writing of the report. The title page should clearly identify any and all competing interests for each author - if there are no competing interests, it should also be declared.

A submission without an abstract is considered incomplete. For all case reports, we require a short (up to 250 words) structured citation-free abstract that provides a succinct summary of the case report under the headings: Purpose, Clinical Features, and Conclusions. Well-written 
abstracts are extremely important for readers, as they are freely available online for all published articles. The abstract is often the only part of the article that is read. Consequently, we encourage use of the maximum word count to summarize the key features of the report. The conclusions of the abstract should mirror exactly the conclusions stated in the text.

Following the abstract, there should be a brief one or two paragraph introduction that explains: 1) the clinical issue or problem being addressed, supported by current citations as appropriate; 2) the purpose for reporting the case; and 3) a statement that consent for publication of the report was obtained in writing from either the patient or her/his power of attorney. The novelty of many submitted case reports is limited, because the manuscript was written with no clear purpose in mind, the focused literature search was incomplete, or because the cited articles were interpreted incorrectly or in a biased manner. Therefore, authors should appraise the relevant background literature critically before writing the article. On a related matter, we discourage such claims as, "we report here, for the first time, a case of ....X or Y", even if the statement is correct. It should be recognized for internal auditing purposes that documentation of unusual cases, adverse events, or nearmisses might be insufficient for publication in the peerreviewed literature. Publishable cases should be original and novel, but they should also contain sufficient data to be of scientific and educational value. Next, this brings us to the issue that, understandably, creates confusion for some authors - patient consent for publication.

Since 2004, it has been part of the Journal's editorial policy that written patient consent is required for publication of all case reports/case series - nevertheless, fewer than half of submitted case reports document that such consent was obtained. The rationale for requiring patient consent to publish these types of articles was clearly outlined in an editorial published at the time this policy of the Journal was implemented. ${ }^{1}$ The requirement is based on the need to protect the patient's right to privacy, since the contents of scientific journals are easily searched and freely accessible to the public. This policy is consistent with the editorial policy statement on patient confidentiality as published by the International Committee of Medical Journal Editors (www.icmje.org), and the policy is being adopted, somewhat gradually, by a number of other medical journals. We appreciate that many hospitals do not have a standard review process for considering patient consent for publication. As it differs from an ethical review to conduct research in humans, most Research Ethics Boards are not involved in this type of review process. Therefore, to assist authors, we recently posted a consent form template for publication that can be downloaded from our article submission platform at: http://cja.edmgr.com.
Authors may be asked to forward a copy of the signed consent form to the editorial office as part of the article submission process. A statement should appear within the text indicating that consent for publication of the article, including related images, was obtained in writing.

Following the brief introduction, the clinical details of the case should be presented sequentially and in sufficient detail that readers will fully grasp the clinical context of the case, from diagnosis - to treatment - to patient outcome. We encourage use of supporting tables and figures, especially for the presentation of physiological variables over time, such as hemodyamic variables and pain scores. A key point may be made by a photograph, radiograph, or ultrasound image. We also encourage use of supporting video clips for online publication as electronic supplementary material (ESM).

Finally, the discussion section (approximately 600-800 words) of the article should provide a clinical interpretation of the case in addition to an explanation of the related clinical decision-making. Again, the relevant literature should be cited carefully, as appropriate. It is important to avoid turning the discussion into a mini review article, as this is not the primary purpose of case reports. The one or two important highlights of the case should be explained clearly, while idle speculation should be avoided completely. The discussion should focus on the case presented, and didactic "textbook" reviews should be avoided. Furthermore, it may be difficult to formulate specific recommendations regarding efficacy or patient safety on the basis of a single case or a small case series; hence, the conclusions of the article must be framed carefully.

Considering the foregoing guidelines, it may be apparent to authors considering submission of case reports that the most important educational element of a given case can be distilled into a focused clinical description of fewer than 800 words. We therefore welcome submission of brief cases presented in the format of a Letter to the Editor, to be considered for publication in the Correspondence section of the Journal. For all published letters, we prefer that the number of authors be limited to no more than three. Published letters may be accompanied by a single figure or image or by a table that has a limited number of columns, rows, and cells. Consent for publication is still required.

In conclusion, we will continue to welcome case reports, and we will continue to publish a limited number in the Journal. However, in our continued effort to enhance the accuracy and clarity of all articles published in the Journal, reporting standards regarding reports of original investigations have become increasingly rigorous. During our editorial peer review process, the novelty and importance of all case reports that advance to external review are considered carefully. The editorial decision regarding publication is based on all of the factors considered in this 
article. We sincerely look forward to having our readers increasingly value the Case Reports section of the Journal.

\section{Les présentations de cas et le Journal : une mise à jour destinée aux auteurs et aux lecteurs}

Tout comme c'est le cas pour nombre de revues médicales scientifiques spécialisées, nous continuons, au fil des années, à recevoir bien plus de présentations de cas et de séries de cas (dans cet article les «présentations de cas » 'case reports') qu'il nous est possible de publier. Nous sommes conscients que les cliniciens souhaitent lire des présentations de cas à la fois bien rédigées, accompagnées de références pertinentes et suffisamment détaillées. En effet, lorsqu'elles rapportent des événements cliniques rares ou inhabituels qui ne peuvent être étudiés ou évalués dans le cadre d'une étude clinique, ces présentations s'ajoutent alors au corpus de nos connaissances cliniques. De plus, certaines revues médicales ont limité le nombre de présentations de cas, voire cessé complètement leur publication en raison de changements en matière de politique rédactionnelle et/ou d'un effet à la baisse sur le facteur d'impact d'une revue (soit la fréquence moyenne de citation d'une revue). Toutefois, étant donné la nature majoritairement clinique de notre lectorat, les présentations de cas bien élaborées qui relatent des expériences cliniques récentes en rapport avec l'anesthésie, la médecine périopératoire, la prise en charge de la douleur et les soins critiques occupent encore une place de choix dans notre contenu rédactionnel. Néanmoins, nous avons remarqué plusieurs problèmes récurrents, lesquels ont souvent pour résultat le rejet de nombreuses présentations de cas soumises et ce, avant même leur évaluation externe. Dans le but de résoudre ces problèmes récurrents, nous avons préparé cet éditorial afin de présenter aux auteurs potentiels et aux lecteurs une vue d'ensemble de la position du Journal en ce qui concerne nos exigences pour qu'une présentation de cas soit évaluée pour publication. Nous présenterons également nos perspectives rédactionnelles quant au contenu jugé convenable pour la publication.

Une condition préalable de base pour toute présentation de cas soumise au Journal est la suivante : elle doit respecter les exigences propres à ce type d'article. Ces exigences sont disponibles en ligne dans la section des Directives aux auteurs (disponibles au: http://cja.edmgr. com). Outre le respect des principes généraux de préparation de l'article spécifiques au Journal, une attention particulière doit être accordée aux points suivants : le respect du nombre maximum de mots (maximum 2000 mots, sans le résumé), une bonne qualité des images, des légendes adaptées et un nombre limité de références pertinentes (10-25). Nous encourageons tout particulièrement les auteurs à s'assurer que le formatage, la grammaire, la syntaxe, l'orthographe et la terminologie scientifique et clinique sont utilisés à bon escient dans leurs articles. Il n'est pas nécessaire que chaque auteur qui écrit et soumet une présentation de cas ait beaucoup de publications à son actif ou qu'il soit un clinicien-chercheur. En revanche, tout auteur soumettant une présentation de cas doit garder à l'esprit qu'une préparation d'article incomplète ou inadéquate pourrait constituer un motif de rejet du manuscrit et ce, avant même qu'il ne soit soumis pour évaluation à l'externe.

La liste des auteurs doit se limiter aux personnes qui ont tenu un rôle direct dans la rédaction et l'interprétation des données présentées. Une longue liste d'auteurs (nous recevons de nombreuses présentations de cas indiquant plus de quatre auteurs) est inappropriée pour la plupart des présentations de cas. Lorsque plusieurs cliniciens-soit plus de deux ou trois-ont participé aux soins cliniques du cas rapporté, mais qu'ils n'ont pas véritablement pris part à la rédaction de la présentation, il faut envisager de remercier séparément ces personnes. Tous les auteurs énumérés doivent avoir contribué de manière substantielle à la rédaction du manuscrit. La page de titre doit identifier clairement les conflits d'intérêt de chaque auteur; s'il n'y a pas de conflit d'intérêt, cela doit également être mentionné.

La soumission d'un manuscrit sans résumé est considérée comme incomplète. Pour toutes les présentations de cas, nous exigeons un bref résumé structuré (jusqu'à 250 mots) sans citation qui propose un résumé succinct de la présentation de cas et est divisé selon les sections suivantes : Objectif, Éléments cliniques et Conclusion. Les résumés bien rédigés sont extrêmement importants pour les lecteurs, étant donné qu'ils sont disponibles gratuitement en ligne pour tout article publié. De plus, le résumé est souvent la seule partie de l'article à être lue. Pour cette raison, nous recommandons aux auteurs de faire usage du nombre maximal de mots pour résumer les éléments clés de leur présentation. La conclusion du résumé doit refléter fidèlement la conclusion de l'article.

À la suite du résumé devrait se trouver une brève introduction d'un ou deux paragraphes expliquant : 1) la situation ou le problème clinique abordé, soutenu dans la mesure du possible par des citations les plus récentes; 2) la raison pour laquelle le cas est présenté; et 3) une déclaration stipulant que le consentement du patient ou de la procuration a été obtenu par écrit pour la publication du cas. L'originalité de plusieurs présentations de cas est limitée parce que le manuscrit a été rédigé sans but clair à l'esprit, parce que la recherche de littérature sur le sujet était incomplète, ou parce que les articles cités ont été interprétés de façon erronée ou biaisée. C'est pourquoi, 
avant de rédiger leur article, les auteurs devraient porter un regard critique sur la littérature préexistante sur le sujet. Par ailleurs, nous décourageons l'emploi d'affirmations telles que : « nous rapportons ici, pour la première fois, un cas de $\mathrm{X}$ ou Y... », même si cette déclaration est correcte. Il importe de reconnaitre que la documentation de cas inhabituels, d'événements indésirables ou d'accidents évités de justesse pourrait ne pas être suffisante pour permettre la publication dans une revue à comité de pairs, et ce en raison de la difficulté de vérification interne des données présentées. Les présentations publiables doivent être originales et innovantes, mais elles doivent également contenir suffisamment de données pour prétendre à une valeur scientifique ou éducationnelle. Ceci nous mène au problème qui génère, à juste titre, beaucoup de confusion chez certains auteurs : le consentement du patient pour la publication.

Depuis 2004, le consentement écrit du patient fait partie des exigences pour la publication de toute présentation de cas ou série de cas dans le cadre de la politique rédactionnelle du Journal; pourtant, moins de la moitié des présentations de cas soumises attestent qu'un tel consentement a été obtenu. À l'époque de la mise en œuvre de cette politique par le Journal, un éditorial avait été publié expliquant clairement pourquoi le Journal exigeait un tel consentement pour la publication de ce type d'article ${ }^{1}$. Cette exigence se fonde sur le besoin de protéger le droit à la vie privée du patient, étant donné que le contenu des revues scientifiques est facile d'accès et disponible au grand public. Cette politique est conforme à l'énoncé de politique rédactionnelle concernant le droit des patients à la confidentialité tel qu'il a été publié par le Comité international des rédacteurs de revues médicales (www. icmje.org), et elle est petit à petit adoptée par plusieurs autres revues médicales. Nous sommes conscients que plusieurs hôpitaux ne disposent pas d'un processus de révision standardisé qui tienne compte du consentement du patient pour la publication. Étant donné que ce genre de consentement ne fait pas partie de la démarche éthique nécessaire pour la recherche chez l'humain, la plupart des comités d'éthique de la recherche ne sont pas impliqués dans ce type de processus de révision. Pour cette raison et dans le but d'aider les auteurs, nous avons récemment publié un modèle de formulaire en ligne de consentement pour publication. Il peut être téléchargé à partir de notre plate-forme de soumission d'article au : http://cja.edmgr. com. Il est possible que le bureau de rédaction du Journal demande aux auteurs un exemplaire du formulaire de consentement signé dans le cadre du processus de soumission du manuscrit. Une déclaration doit apparaître dans le texte indiquant que le consentement pour la publication de l'article, y compris des images connexes, a été obtenu par écrit.
À la suite de la brève introduction, les détails cliniques du cas doivent être présentés de façon chronologique et suffisamment détaillée afin que le lecteur puisse saisir pleinement le contexte clinique du cas, du diagnostic au devenir du patient, en passant par le traitement. Nous recommandons l'utilisation de tableaux et de figures à l'appui, particulièrement lors de la présentation de variables physiologiques dans le temps, comme par exemple des variables hémodynamiques ou des scores de douleur. Un point clé peut être souligné par une photographie, une radiographie, ou une échographie. Nous appuyons également l'emploi de clips vidéos pertinents pour la publication en ligne en format ESM (matériel électronique supplémentaire).

Enfin, la section de discussion (environ 600 à 800 mots) de l'article doit présenter une interprétation clinique du cas en plus d'une explication de la prise de décision clinique associée. La littérature pertinente devrait à nouveau être citée avec soin et être bien adaptée au propos. Il est important d'éviter de faire de la section de discussion un bref article de revue, étant donné qu'il ne s'agit pas de l'objectif premier d'une présentation de cas. Les quelques points saillants du cas doivent être clairement expliqués, et les conjectures futiles doivent absolument être bannies. La discussion doit se concentrer sur le cas présenté, et les revues didactiques de style 'manuel scolaire' doivent être évitées. De plus, il peut être difficile de formuler des recommandations spécifiques concernant l'efficacité ou la sécurité pour le patient d'un traitement donné sur la base d'un seul cas ou d'une petite série de cas; dès lors, il importe de bien mettre en contexte les conclusions de l'article.

$\mathrm{Au}$ vu des directives énumérées ci-dessus, il devient évident pour les auteurs envisageant la soumission d'une présentation de cas que l'élément éducationnel le plus important d'un cas donné peut être condensé en une description clinique ciblée de moins de 800 mots. C'est pourquoi nous sommes ouverts à la soumission de cas brefs présentés sous forme de Lettres à la rédaction, qui pourraient être publiées dans la section de Correspondance du Journal. Pour les lettres publiées, nous préférons que le nombre d'auteurs se limite à trois au maximum. Les lettres publiées peuvent être accompagnées d'une seule figure ou image ou d'un tableau avec un nombre limité de colonnes, de lignes et de cellules. Le consentement à la publication est également requis pour ce format de publication.

En conclusion, nous continuerons d'accepter la soumission de présentations de cas, et nous continuerons d'en publier un nombre limité dans le Journal. Toutefois, conformément à nos efforts soutenus pour améliorer la précision et la clarté de tous les articles publiés dans le Journal, les normes de rédaction des comptes-rendus de recherches originales sont de plus en plus strictes. Pendant 
notre processus rédactionnel d'évaluation par les pairs, l'originalité et l'importance de toutes les présentations de cas qui progressent jusqu'à l'évaluation à l'externe sont examinées avec soin. La décision de la rédaction de publier ou non se fonde sur tous les facteurs mentionnés dans cet article. Nous nous réjouissons à l'idée que nos lecteurs apprécieront davantage la section des Présentations de cas du Journal.
Competing interests None declared.

\section{Reference}

1. Bevan JC, Hardy JF. Permission to publish case reports/case series. Can J Anesth 2004; 51: 861-6. 